Applied and

NISTIR 89-4143

Computational

Mathematics

Division

Center for Computing and Applied Mathematics

\title{
Effect of Anisotropic Thermal Conductivity on the Morphological Stability of a Binary Alloy
}

S.R. Coriell, G.B. McFadden, and R.F. Sekerka

\author{
August 1989
}

\section{U.S. DEPARTMENT OF COMMERCE}

National Institute of Standards and Technology

Gaithersburg, MD 20899 



\title{
Effect of Anisotropic Thermal Conductivity on the Morphological Stability of a Binary Alloy
}

\author{
S.R. Coriell and G. B. McFadden \\ National Institute of Standards and Technology \\ Gaithersburg, MD 20899, USA \\ and \\ R.F Sekerka* \\ Departments of Physics and Mathematics \\ Carnegie Mellon University \\ Pittsburgh, PA 15213, USA
}

\begin{abstract}
We perform a linear morphological stability analysis of a planar interface during unidirectional solidification of a binary alloy for the case of a crystal having an anisotropic thermal conductivity. We calculate a dispersion relation which shows that the onset of instability depends on the orientation of the growth direction with respect to principal crystallographic axes and on the orientation of the wavevector of the perturbation. The onset of instability can be either oscillatory (travelling waves) or non-oscillatory in time. For growth along a principal axis of the crystal there is an exchange of stabilities, and the onset of instability is non-oscillatory. For a uniaxial crystal, we explore the dispersion relation in detail, and give numerical results for the case of an alloy of 0.78 at\% bismuth in tin. For low growth velocities the onset of instability is nonoscillatory and occurs for perturbations having a wavevector that lies along a principal crystallographic axis.
\end{abstract}

"Consultant, National Institute of Standards and Technology 


\section{Introduction}

The theory of morphological stability, which has been with us now for over 25 years [1], has been used to understand the conditions for the stability of a planar interface during unidirectional solidification of a dilute binary alloy at constant velocity $V$. Experimental tests of this theory abound in the literature, and many of these pertain to cubic materials for which there is no anisotropy of thermal conductivity . For non-cubic crystals, however, the thermal conductivity will generally be anisotropic and the effect of this anisotropy on morphological stability theory is not well understood. Indeed, one of the most popular low melting point materials for experimental studies has been tin $[2,3,4,5,6,7,8]$, which is uniaxial, having a thermal conductivity $k_{\|}=0.460 \mathrm{~J} /(\mathrm{cm} \cdot K \cdot s)$ along the axis of symmetry, and $k_{\perp}=0.662 \mathrm{~J} /(\mathrm{cm} \cdot K \cdot s)$ along any direction perpendicular to the axis of symmetry [9]. Moreover, tin has been selected as a prototype material for the study of morphological stability under microgravity conditions by using the MEPHISTO system [10].

One might think that the standard morphological stability results could be modified simply for an anisotropic crystal by replacing the thermal conductivity of the solid with the thermal conductivity of the anisotropic crystal in some appropriate direction; however, this begs the question of what direction to use as well as the question of the effect of components of the heat flux that are not necessarily parallel to the temperature gradient in an anisotropic crystal. In fact, our analysis, for crystal growth in a given direction, will necessitate exploration of the conditions for marginal stability as a function of both the magnitude and direction of the wavevector of infinitesimal sinusoidal perturbations of the unperturbed planar interface. We shall find that for some of these directions, the onset of morphological instability can even be oscillatory in time. For growth along a principal crystallographic direction there is an exchange of stabilities and the onset of instability is non-oscillatory. We explore the case of a uniaxial crystal in detail. 


\section{Theory}

We perform a linear stability analysis of an initially planar crystal-melt interface for a crystal with anisotropic thermal conductivity. For simplicity we neglect anisotropy of other properties, such as surface tension and interface kinetics, which have been treated previously $[11,12,13]$ and are even important for cubic materials. The planar crystal-melt interface is assumed to be moving in the $z$ direction at constant velocity $V$ with respect to the crystal. We choose a Cartesian coordinate system $(x, y, z)$ which is fixed with respect to the unperturbed planar interface located at $z=0$. The perturbed interface, $z=h(x, y, t)$, is assumed to be periodic in both the $x$ and $y$ directions.

The solute field, $c$, then satisfies

$$
c_{t}=D \nabla^{2} c+V c_{z}, \text { for } z>h(x, y, t)
$$

where $D$ is the solute diffusivity and $\nabla^{2}$ is the Laplacian; subscripts $x, y, z$, or $t$ on the dependent variables indicate partial derivatives. In the liquid, we assume that the temperature field, $T_{L}$, in the thermal steady-state approximation [14], satisfies

$$
\nabla^{2} T_{L}=0, \text { for } z>h(x, y, t)
$$

In the same approximation, the temperature field $T_{S}$ in the crystal satisfies

$$
\sum_{i, j=1}^{3} k_{i j} \frac{\partial^{2} T_{S}}{\partial x_{i} \partial x_{j}}=0, \text { for } z<h(x, y, t)
$$

where $k_{i j}=k_{j i}$ is the (symmetric, positive-definite) thermal conductivity tensor in the crystal. In the summation we have used the notation $x_{1}=x, x_{2}=y$, and $x_{3}=z$. We will refer to $k_{11}$ as $k_{x x}$, and so on.

The boundary conditions at the crystal-melt interface $z=h(x, y, t)$ are

$$
\begin{gathered}
T_{S}=T_{L}=T_{M}+m c-T_{M} \Gamma \mathcal{K} \\
-D\left(c_{z}-h_{x} c_{x}-h_{y} c_{y}\right)=\left(V+h_{t}\right)(1-k) c \\
\left(-k_{L} \nabla T_{L}-q_{S}\right) \cdot n=L_{V}\left(V+h_{t}\right) n_{z} .
\end{gathered}
$$


Here the distribution coefficient $k$ relates the interface concentration, $c$, in the liquid, to that, $c_{S}$, in the solid via $c_{S}=k c ; L_{V}$ is the latent heat of fusion per unit volume (we take the densities of crystal and melt to be equal); $m$ is the liquidus slope; $k_{L}$ is the thermal conductivity in the liquid; $T_{M}$ is the melting point of the pure material; $\Gamma$ is the capillary length associated with the crystal-melt surface energy; and $\mathcal{K}$ is the mean curvature of the interface. The heat flux vector, $q_{S}$, in the crystal has components given by $\left(q_{S}\right)_{i}=$ $-\sum_{j=1}^{3} k_{i j} \partial T_{S} / \partial x_{j}$. The unit normal to the interface is given by

$$
\boldsymbol{n}=\frac{\left(-h_{x},-h_{y}, 1\right)}{\sqrt{1+h_{x}^{2}+h_{y}^{2}}}
$$

The far-field boundary conditions are

$$
\begin{gathered}
c \rightarrow c_{\infty}, \quad \text { as } z \rightarrow \infty, \\
\partial T_{L} / \partial z \rightarrow G_{L}, \quad \text { as } z \rightarrow \infty,
\end{gathered}
$$

and

$$
\partial T_{S} / \partial z \rightarrow G_{S} \text { as } z \rightarrow-\infty
$$

the value for $G_{S}$ is actually set by the heat flux boundary condition in order to allow steadystate growth with the given velocity: $k_{z z} G_{S}-k_{L} G_{L}=L_{V} V$.

\subsection{Planar Solution}

The planar solution is given by $h^{(0)}(x, y, t)=0$ and

$$
\begin{gathered}
c^{(0)}(x, y, z, t)=c_{\infty}\left\{1+\frac{(1-k)}{k} \exp (-V z / D)\right\} \\
T_{L}^{(0)}(x, y, z, t)=T_{M}+\frac{m c_{\infty}}{k}+G_{L} z \\
T_{S}^{(0)}(x, y, z, t)=T_{M}+\frac{m c_{\infty}}{k}+G_{S} z
\end{gathered}
$$




\subsection{Linear Stability Analysis}

We write

$$
\left(\begin{array}{c}
c(x, y, z, t) \\
T_{L}(x, y, z, t) \\
T_{S}(x, y, z, t) \\
h(x, y, t)
\end{array}\right)-\left(\begin{array}{c}
c^{(0)}(z) \\
T_{L}^{(0)}(z) \\
T_{S}^{(0)}(z) \\
0
\end{array}\right)=\left(\begin{array}{c}
c^{(1)}(x, y, z, t) \\
T_{L}^{(1)}(x, y, z, t) \\
T_{S}^{(1)}(x, y, z, t) \\
h^{(1)}(x, y, t)
\end{array}\right)=\left(\begin{array}{c}
\hat{c}(z) \\
\hat{T}_{L}(z) \\
\hat{T}_{S}(z) \\
\delta
\end{array}\right) \exp \left(\sigma t+i \omega_{x} x+i \omega_{y} y\right)
$$

where the perturbed quantities (superscript " 1 ") are assumed to be small.

The solution to the perturbed solute equation is unchanged from the standard case [15]:

$$
\hat{c}(z)=\hat{c}(0) \exp (r z)
$$

where

$$
r=-\frac{V}{2 D}-\sqrt{\left(\frac{V}{2 D}\right)^{2}+\omega^{2}+\frac{\sigma}{D}}
$$

and

$$
\hat{c}(0)\{D r+V(1-k)\}=\delta\left\{V k G_{c}-\sigma(1-k) \frac{c_{\infty}}{k}\right\} .
$$

Here $\omega^{2}=\omega_{x}^{2}+\omega_{y}^{2}$ and $G_{c}=c_{\infty}(V / D)(k-1) / k$.

Similarly, the perturbed temperature in the liquid has the usual form,

$$
\hat{T}_{L}(z)=\hat{T}_{L}(0) \exp (-\omega z)
$$

where the constant $\hat{T}_{L}(0)$ is determined from the interface boundary conditions.

The perturbed temperature in the solid satisfies

$$
k_{z z} \frac{\partial^{2} \hat{T}_{S}}{\partial z^{2}}+2 i Q \frac{\partial \hat{T}_{S}}{\partial z}-P \hat{T}_{S}=0
$$

where

$$
Q=\omega_{x} k_{x z}+\omega_{y} k_{y z}
$$

and

$$
P=k_{x x} \omega_{x}^{2}+2 k_{x y} \omega_{x} \omega_{y}+k_{y y} \omega_{y}^{2}
$$


The solution to Eq. (5) can be written in the form

$$
\hat{T}_{S}(z)=\hat{T}_{S}(0) \exp \left(r_{S} z\right)
$$

where

$$
r_{S}=-\frac{i Q}{k_{z z}}+\frac{1}{k_{z z}} \sqrt{P k_{z z}-Q^{2}} .
$$

The expression inside the radical can be rewritten in the form

$$
P k_{z z}-Q^{2}=A \omega_{x}^{2}+2 B \omega_{x} \omega_{y}+C \omega_{y}^{2}
$$

where

$$
\begin{aligned}
& A=k_{x x} k_{z z}-k_{x z}^{2}, \\
& B=k_{z z} k_{x y}-k_{x z} k_{y z}, \\
& C=k_{y y} k_{z z}-k_{y z}^{2} .
\end{aligned}
$$

The expression (7) is a positive-definite quadratic form in $\omega_{x}$ and $\omega_{y}$, since we have $A>0$, $C>0$, and $A C-B^{2}>0$. The first two inequalities follow from the fact that $A$ and $C$ are given by minors of the positive-definite tensor $\left\{k_{i j}\right\}$, and the third inequality follows from the identity $A C-B^{2}=k_{z z} \operatorname{det}\left(k_{i j}\right)$, which can be verified by expanding the determinant. From these considerations it follows that the real part of $r_{S}$ is positive, so that $\hat{T}_{S}$ decays as $z \rightarrow-\infty$.

Linearization of the heat flux boundary condition proceeds as follows. Written out more fully, the nonlinear equation is

$$
\begin{array}{r}
L_{V}\left(V+h_{t}\right)+k_{L}\left(\frac{\partial T_{L}}{\partial z}-h_{x} \frac{\partial T_{L}}{\partial x}-h_{y} \frac{\partial T_{L}}{\partial y}\right)=\left(k_{x z} \frac{\partial T_{S}}{\partial x}+k_{y z} \frac{\partial T_{S}}{\partial y}+k_{z z} \frac{\partial T_{S}}{\partial z}\right) \\
-h_{x}\left(k_{x x} \frac{\partial T_{S}}{\partial x}+k_{x y} \frac{\partial T_{S}}{\partial y}+k_{x z} \frac{\partial T_{S}}{\partial z}\right) \\
-h_{y}\left(k_{x y} \frac{\partial T_{S}}{\partial x}+k_{y y} \frac{\partial T_{S}}{\partial y}+k_{y z} \frac{\partial T_{S}}{\partial z}\right) .
\end{array}
$$

The linearized form of Eq. (11) is therefore

$$
L_{V} h_{t}^{(1)}+k_{L} \frac{\partial T_{L}^{(1)}}{\partial z}=\left(k_{x z} \frac{\partial T_{S}^{(1)}}{\partial x}+k_{y z} \frac{\partial T_{S}^{(1)}}{\partial y}+k_{z z} \frac{\partial T_{S}^{(1)}}{\partial z}\right)-h_{x}^{(1)} k_{x z} G_{S}-h_{y}^{(1)} k_{y z} G_{S}
$$


or

$$
\sigma L_{V} \delta-k_{L} \omega \hat{T}_{L}=k_{z z} r_{S} \hat{T}_{S}+i Q\left(\hat{T}_{S}-G_{S} \delta\right)
$$

where all quantities are understood to be evaluated at $z=0$. At $z=0$ we also have

$$
\hat{T}_{L}+G_{L} \delta=\hat{T}_{S}+G_{S} \delta=m\left(\hat{c}+G_{c} \delta\right)-T_{M} \Gamma \omega^{2} \delta
$$

Equations (3c), (13), and (14) constitute a set of homogeneous linear equations in the unknowns $\hat{c}, \hat{T}_{L}, \hat{T}_{S}$, and $\delta$; they have a nontrivial solution only if

$$
\begin{aligned}
& \frac{i Q G_{S}+\sigma L_{V}}{k_{L} \omega+\sqrt{P k_{z z}-Q^{2}}}+\frac{k_{L} \omega G_{L}}{k_{L} \omega}+\sqrt{P k_{z z}-Q^{2}} G_{S}= \\
&-T_{M} \Gamma \omega^{2}+m k_{c}-Q^{2} \\
& R-(1-2 k) V / 2 D
\end{aligned}
$$

where

$$
R=\sqrt{\left(\frac{V}{2 D}\right)^{2}+\omega^{2}+\frac{\sigma}{D}}
$$

Eq. (15) can be viewed as a dispersion relation for $\sigma$ as a function of $\omega_{x}$ and $\omega_{y}$ for a given set of material properties and growth conditions. For isotropic thermal conductivity this relation reduces to previously-obtained results [14]. In fact, the term in $G_{L}$ and $G_{S}$ has the familiar form of a conductivity-weighted average of $G_{L}$ with $k_{L}$ and $G_{S}$ with an effective conductivity $k_{E}=\sqrt{P k_{z z}-Q^{2}} / \omega$, which is independent of the magnitude (but not the direction) of $\omega$ as can be seen from Eq. (7).

If the real part of $\sigma$ is negative for all values of $\omega_{x}$ and $\omega_{y}$, the planar interface is stable. By eliminating $\sigma$ using Eq. (16), Eq. (15) can be expressed as a cubic polynomial in the variable $R$ and from the roots of this polynomial that have $\operatorname{Real}(R) \geq 0$ one can deduce $\sigma$ as a function of $\omega_{x}$ and $\omega_{y}$. In the next section we analyze this dispersion relation for a crystal with uniaxial symmetry. 


\section{Analysis of the Dispersion Relation for a Uniaxial Crystal}

For a uniaxial crystal, such as tin, the conductivity tensor in a coordinate system aligned with the principal axes of the crystal can be written in the form

$$
\left\{k_{i j}\right\}=\left(\begin{array}{ccc}
k_{\perp} & 0 & 0 \\
0 & k_{\perp} & 0 \\
0 & 0 & k_{\|}
\end{array}\right),
$$

where $k_{\perp}$ and $k_{\|}$are generally unequal. The relation between this system of axes (see Fig. 1) and the $(x, y, z)$ axes can be described by means of the orthogonal matrix

$$
\mathcal{R}=\left(\begin{array}{ccc}
1 & 0 & 0 \\
0 & \cos \theta & \sin \theta \\
0 & -\sin \theta & \cos \theta
\end{array}\right)
$$

which produces a rotation by an angle $\theta$ about the $x$ axis. This suffices to describe the general case because at least one principal crystallographic axis corresponding to $k_{\perp}$ can always be taken to lie along the $x$ axis because of degeneracy in the plane perpendicular to the uniaxial principal axis. The conductivity tensor transforms according to

$$
\mathcal{R}^{T}\left(\begin{array}{ccc}
k_{\perp} & 0 & 0 \\
0 & k_{\perp} & 0 \\
0 & 0 & k_{\|}
\end{array}\right) \mathcal{R}=\left(\begin{array}{ccc}
k_{\perp} & 0 & 0 \\
0 & k_{y y} & k_{y z} \\
0 & k_{y z} & k_{z z}
\end{array}\right)
$$

where

$$
\begin{aligned}
& k_{y y}=k_{\|} \sin ^{2} \theta+k_{\perp} \cos ^{2} \theta \\
& k_{z z}=k_{\|} \cos ^{2} \theta+k_{\perp} \sin ^{2} \theta \\
& k_{y z}=\left(k_{\perp}-k_{\|}\right) \cos \theta \sin \theta
\end{aligned}
$$

which leads to $A=k_{\perp} k_{z z}, B=0$ and $C=k_{\perp} k_{\|}$. We write the wavevector in the form $\left(\omega_{x}, \omega_{y}\right)=\omega(\cos \alpha, \sin \alpha)$ to obtain

$$
P=k_{\perp} \omega^{2} \cos ^{2} \alpha+\omega^{2} \sin ^{2} \alpha\left(k_{\|} \sin ^{2} \theta+k_{\perp} \cos ^{2} \theta\right),
$$




$$
Q=\left(k_{\perp}-k_{\|}\right) \omega \sin \alpha \cos \theta \sin \theta
$$

the effective conductivity becomes

$$
k_{E}(\theta, \alpha)=\sqrt{k_{\perp} k_{z z}(\theta) \cos ^{2} \alpha+k_{\perp} k_{\|} \sin ^{2} \alpha}
$$

We rewrite Eq. (15) in the form

$$
\frac{i Q G_{S}+\sigma L_{V}}{k_{L} \omega+k_{E} \omega}+G_{L} F(\theta, \alpha)=-T_{M} \Gamma \omega^{2}+m G_{c} \frac{(R-V / 2 D)-\sigma / V}{R-(1-2 k) V / 2 D}
$$

where

$$
F(\theta, \alpha)=\frac{1}{1+k_{E}(\theta, \alpha) / k_{L}}\left(1+\left\{1+\frac{V L_{V}}{k_{L} G_{L}}\right\} \frac{k_{E}(\theta, \alpha)}{k_{z z}(\theta)}\right) .
$$

Clearly $k_{z z}(\theta)$ and $k_{E}(\theta, \alpha)$ are symmetric about the points $\theta=0$ and $\theta=\pi / 2$, and about $\alpha=0$ and $\alpha=\pi / 2$. For $\theta=0$ we note that $k_{E}(0, \alpha)$ and $F(0, \alpha)$ are both independent of $\alpha$.

In analyzing Eq. (17), the following questions are of interest: for crystal growth in a given crystallographic direction (fixed $\theta$ ), for what value of $\alpha$ does the onset of instability occur? At this onset, is the imaginary part of $\sigma$ non-zero, so that the onset is oscillatory in time (travelling waves)? How do these results change as a function of $\theta$ ?

We first present some numerical results for dilute alloys of 0.78 at\% bismuth in tin (for thermophysical properties given in Table I) and then establish some general features of Eq. (17).

In Figs. 2 and 3 we plot the marginal values of $G_{L}$ and $\sigma_{i}=\operatorname{Im}(\sigma)$, as functions of $\alpha$ for a growth velocity of $5 \mu \mathrm{m} / \mathrm{s}$ and various values of $\theta$; these marginal values are calculated by maximizing $G_{L}$ over $\omega$ for fixed $\alpha$. Observe, for any $\theta$, that the onset of instability occurs at $\alpha=0$, and with $\sigma_{i}=0$. For $\theta=0$ and $\theta=\pi / 2, \sigma_{i}=0$ for all values of $\alpha$; these values of $\theta$ correspond to cases for which the growth direction is along a principal crystallographic axis, a case that we shall analyze later. We have plotted these figures only for positive values of $\alpha$ and $\theta$ lying between zero and $\pi / 2$; changing the sign of either $\theta$ or $\alpha$ changes the sign of $\sigma_{i}$ but $G_{L}$ is unaffected. We observe that the critical value of $G_{L}$ at the onset of instability varies by about $25 \%$ with crystallographic orientation; this should be viewed in light of an anisotropy in thermal conductivity of $44 \%$ for tin. 
In Figs. 4 and 5 we again plot the marginal values of $G_{L}$ and $\sigma_{i}$ as functions of $\alpha$ for a much larger growth velocity, $V=30 \mathrm{~cm} / \mathrm{s}$. The features of these curves are similar to those of Figs. 2 and 3, except that the onset of instability now occurs at $\alpha=\pi / 2$ and for a non-zero value of $\sigma_{i}$. Therefore the onset of instability is oscillatory at this higher velocity for $0<\theta<\pi / 2$. We note that this velocity is approaching the conditions for absolute stability, and the marginal temperature gradients change extremely rapidly with velocity; therefore the apparent anisotropy displayed in Fig. 4 would be very difficult to observe experimentally.

In order to better understand the features of these curves, we now analyze Eq. (17). For the range of values used in the computations, the product $G_{L} F(\theta, \alpha)$ appears to be nearly independent of $\theta$ and $\alpha$, so the anisotropy of $G_{L}$ can be understood approximately in terms of the anisotropy of $F$. For fixed $\theta \neq 0$ we consider the extrema of $F(\theta, \alpha)$ as $\alpha$ varies. We have

$$
\frac{\partial F}{\partial \alpha}=\frac{1}{\left\{1+k_{E}(\theta, \alpha) / k_{L}\right\}^{2}}\left(\frac{1}{k_{z z}}\left\{1+\frac{V L_{V}}{k_{L} G_{L}}\right\}-\frac{1}{k_{L}}\right) \frac{\partial k_{E}}{\partial \alpha}
$$

and

$$
k_{E} \frac{\partial k_{E}}{\partial \alpha}=k_{\perp}^{2}\left(\frac{k_{\|}}{k_{\perp}}-1\right) \sin ^{2} \theta \sin \alpha \cos \alpha .
$$

Therefore, aside from the case in which $\partial F / \partial \alpha$ vanishes identically (which corresponds to $\left.G_{S}=G_{L}\right)$, we have extrema only at $\alpha=0$ and $\alpha=\pi / 2 . F(\theta, \alpha)$ is therefore an increasing function of $\alpha$ if

$$
\left(\frac{k_{\perp}}{k_{z z}}\left\{1+\frac{V L_{V}}{k_{L} G_{L}}\right\}-\frac{k_{\perp}}{k_{L}}\right)\left(\frac{k_{\|}}{k_{\perp}}-1\right)>0
$$

in which case $\alpha=0$ corresponds to a minimum and $\alpha=\pi / 2$ is a maximum. If this expression is negative, then the minimum and maximum are interchanged. This explains the difference in appearance in Figs. 2 and 4, because the term $V L_{V} /\left(k_{L} G_{L}\right)$ is negligible for $V=5 \mu \mathrm{m} / \mathrm{s}$, but becomes large enough at $V=30 \mathrm{~cm} / \mathrm{s}$ to interchange the values of $\alpha$ at which the maxima and minima of $F$ occur. Note that a maximum of $F$ corresponds to a minimum of $G_{L}$, and vice versa, and also that the extrema of $F$ correspond to the extrema of $k_{E}$.

We next consider the extrema of $F(\theta, \alpha)$ as $\theta$ varies for fixed $\alpha$. A short calculation shows that

$$
\frac{\partial F}{\partial \theta}=-C_{1} \frac{\partial k_{z z}}{\partial \theta}
$$


where $C_{1}$ is a positive factor. Since

$$
\frac{\partial k_{z z}}{\partial \theta}=2\left(k_{\perp}-k_{\|}\right) \cos \theta \sin \theta
$$

we conclude that $\theta=0$ and $\theta=\pi / 2$ are extrema of $F$, with $\theta=0$ being a maximum and $\theta=\pi / 2$ a minimum for $k_{\perp}>k_{\|}$.

For either $\theta=0$ or $\theta=\pi / 2$, we have $Q=0$ which (since always $k_{x z}=0$ for our choice of axes) is a manifestation of $k_{y z}=0$. Both $\theta=0$ and $\theta=\pi / 2$ correspond to orientations for which $x, y, z$ are principal axes of the crystal. Moreover, if $Q=0$, we can deduce from Eq. (15) that $\sigma_{i}=0$, because the problem is then of the same form as that in previous treatments [16] for isotropic conductivity, for which the principle of exchange of stabilities has been established.

\section{Discussion}

We have carried out a linear morphological stability analysis for anisotropic thermal conductivity in the crystal, resulting in the dispersion relation given by Eq. (15). For fixed growth conditions and thermophysical properties, this dispersion relation can be considered to be an equation for $\sigma$ as a function of both magnitude and direction of the wavevector (through $\omega_{x}$ and $\omega_{y}$, or alternatively through $\omega$ and $\alpha$ ) as well as the orientation of the growth direction relative to the crystal axes, which requires the specification of two parameters for a crystal of low symmetry. If the growth direction coincides with a principal crystallographic direction, there is an exchange of stabilities, and the onset of instability is non-oscillatory. For other growth directions, the onset of instability might or might not be non-oscillatory.

For a uniaxial crystal it is sufficient to specify only the angle $\theta$ that the axis of symmetry makes with the growth direction, and the resulting dispersion relation is given by Eq. (17). We analyze this dispersion relation numerically for the case of an alloy of 0.78 at\% bismuth in tin, with results given in Figs. 2-5. For low growth velocities, the onset of instability occurs with $\sigma_{i}=0$ for perturbations with wavevector along a principal axis of the crystal that lies in the plane of the unperturbed interface. For high growth velocities, the onset of instability 
occurs for perturbations with wavevector perpendicular to the principal axis of the crystal that lies in the plane of the unperturbed interface, and with $\sigma_{i} \neq 0$ for orientations in the range $0<\theta<\pi / 2$. For other uniaxial crystals, the relative conductivities might be such that the inequality in Eq. (18) might be reversed, resulting in an onset of instabilities at low velocities that is oscillatory in time.

For a crystal of general anisotropy, the appropriate dispersion relation is Eq. (15), which is difficult to analyze. For low velocities (constitutional supercooling regime), however, an approximate analysis is tractible since $R \approx \omega$ and $T_{M} \Gamma \omega^{2} \ll m G_{c}$ at the onset of instability. To this approximation, the real and imaginary parts of Eq. (15) for $\sigma_{r}=0$ give the simple results

$$
\frac{k_{L} G_{L}+k_{E} G_{S}}{k_{L}+k_{E}}=m G_{c}
$$

and

$$
\sigma_{i}=\frac{-V Q}{k_{z z}+k_{E}}
$$

For instance, for the conditions corresponding to Figs. 2 and 3, with $\alpha=\pi / 2$ and $\theta=\pi / 4$, the numerical results are $G_{L}=175 \mathrm{~K} / \mathrm{cm}$ and $\sigma_{i}=-1.70 \mathrm{~s}^{-1}$, and those calculated from Eqns. (19) and (20) are $G_{L}=186 \mathrm{~K} / \mathrm{cm}$ and $\sigma_{i}=-1.78 \mathrm{~s}^{-1}$.

Finally, we note that $k_{E}$ can be written in the form

$$
k_{E}=\sqrt{A \cos ^{2} \alpha+2 B \cos \alpha \sin \alpha+C \sin ^{2} \alpha}
$$

where $A, B$ and $C$ are given by Eqs. (8) - (10). It is easy to determine that its maximum and minimum values are

$$
k_{E}^{ \pm}=\sqrt{\frac{1}{2}(A+C) \pm \frac{1}{2} \sqrt{(A-C)^{2}+4 B^{2}}} .
$$

Then writing Eq. (19) in the form

$$
G_{L}\left[\frac{k_{L}+k_{E}\left(G_{S} / G_{L}\right)}{k_{L}+k_{E}}\right]=m G_{c}
$$

and recognizing that a maximum value of $G_{L}$ will result from a minimum value of the term in braces, we conclude that for $G_{L}>G_{S}, k_{E}^{+}$is applicable, whereas for $G_{L}<G_{S}$, $k_{E}^{-}$is applicable in determining the onset of instability. 


\section{Acknowledgements}

We are grateful to L. N. Brush, S. H. Davis, J.-J. Favier, B. T. Murray, A. Rouzaud, and R. J. Schaefer for helpful discussions. This work was conducted with the support of the Microgravity Science and Applications Division of the National Aeronautics and Space Administration, and the Defense Advanced Research Projects Agency. One of us (RFS) received partial support from the National Science Foundation under Grant DMR84-09397. 


\section{References}

[1] W. W. Mullins and R. F. Sekerka, J. Appl. Phys. 35 (1964) 444.

[2] B. Chalmers, Principles of Solidification, (Wiley, New York, 1964), Ch. 5.

[3] D. Walton, W. A. Tiller, J. W. Rutter, and W. C. Winegard, Trans. AIME 203 (1955) 1023.

[4] H. Biloni and G. F. Bolling, Trans. AIME 227 (1963) 1351.

[5] J. J. Kramer, G. F. Bolling, and W. A. Tiller, Trans. AIME 227 (1963) 374.

[6] H. Biloni, R. DiBella, and G. F. Bolling, Trans. AIME 239 (1967) 2012.

[7] J. C. Warner and J. D. Verhoeven, Metall. Trans. 4 (1973) 1255.

[8] K. Suzuki, A. Hikata, and C. Elbaum, Phys. Rev. Lett. 59 (1987) 2686.

[9] Y. S. Touloukian, R. W. Powell, C. Y. Ho, and P. G. Klemens, Thermophysical Properties of Matter. Vol. 1. Thermal Conductivity. Metallic Elements and Alloys (IFI/Plenum, New York, 1970), pp. 408-409.

[10] J.-J. Favier and A. Rouzaud, Adv. Space Res. 6 (1986) 111.

[11] S. R. Coriell and R. F. Sekerka, J. Crystal Growth 34 (1976) 157.

[12] G. W. Young, S. H. Davis, and K. Brattkus, J. Crystal Growth 83 (1987) 560.

[13] G. B. McFadden, S. R. Coriell, and R. F. Sekerka, J. Crystal Growth 91 (1988) 180.

[14] S. R. Coriell, G. B. McFadden, and R. F. Sekerka, Ann. Rev. Mater. Sci. 15 (1985) 119.

[15] R. F. Sekerka, in: Crystal Growth, Ed. H. S. Peiser, (Pergammon, Oxford, 1967) p. 691.

[16] S. R. Coriell, G. B. McFadden, P. W. Voorhees, and R. F. Sekerka, J. Crystal Growth 82 (1987) 295. 


\section{Table I}

Values used in numerical computations

\begin{tabular}{lll}
\hline liquid diffusion coefficient & $D$ & $1.8 \cdot 10^{-5} \mathrm{~cm}^{2} / \mathrm{s}$ \\
liquid thermal conductivity & $k_{L}$ & $0.303 \mathrm{~J} /(\mathrm{cm} \cdot K \cdot \mathrm{s})$ \\
solid thermal conductivities & $k_{\|}$ & $0.460 \mathrm{~J} /(\mathrm{cm} \cdot K \cdot \mathrm{s})$ \\
& $k_{\perp}$ & $0.662 \mathrm{~J} /(\mathrm{cm} \cdot K \cdot \mathrm{s})$ \\
distribution coefficient & $k$ & 0.28 \\
liquidus slope & $m$ & $-2.28 \mathrm{~K} / \mathrm{at} \%$ \\
capillary parameter & $T_{M} \Gamma$ & $8.6 \cdot 10^{-6} \mathrm{~cm} \cdot K$ \\
heat of fusion & $L_{V}$ & $418.0 \mathrm{~J} / \mathrm{cm}^{3}$ \\
\hline
\end{tabular}




\section{Figure Captions}

Figure 1. Drawing showing the orientation of the growth direction, $z$, relative to the principal axes $x_{c}, y_{c}$, and $z_{c}$ of a uniaxial crystal having thermal conductivity $k_{\|}$along $z_{c}$ and $k_{\perp}$ perpendicular to $z_{c}$. Without loss of generality we choose $x$ to lie along $x_{c}$ so that the growth direction can be completely specified by the angle $\theta$. The angle $\alpha$ specifies the direction of the wavevector of a perturbation relative to the $x$ axis.

Figure 2. Marginal values of the temperature gradient $G_{L}$ as a function of $\alpha$ for various growth directions $\theta$ for an alloy of 0.78 at\% bismuth in tin growing at $5 \mu \mathrm{m} / \mathrm{s}$. The interface is stable for values of $G_{L}$ above the given curves, so the onset of instability occurs for $\alpha=0$.

Figure 3. Imaginary part of $\sigma$ versus $\alpha$ for the same conditions as Fig. 2. For $\theta=0$ and $\theta=\pi / 2$, which are principal crystallographic directions, $\sigma_{i}=0$. Note also that $\sigma_{i}=0$ at the onset of instability, which takes place at $\alpha=0$.

Figure 4. Marginal values of the temperature gradient $G_{L}$ as a function of $\alpha$ for various growth directions $\theta$ for an alloy of 0.78 at $\%$ bismuth in tin growing at $30 \mathrm{~cm} / \mathrm{s}$. The interface is stable for values of $G_{L}$ above the given curves, so the onset of instability occurs for $\alpha=\pi / 2$.

Figure 5. Imaginary part of $\sigma$ versus $\alpha$ for the same conditions as Fig. 4. For $\theta=0$ and $\theta=\pi / 2$, which are principal crystallographic directions, $\sigma_{i}=0$. Note also that $\sigma_{i} \neq 0$ at the onset of instability, which takes place at $\alpha=\pi / 2$. 


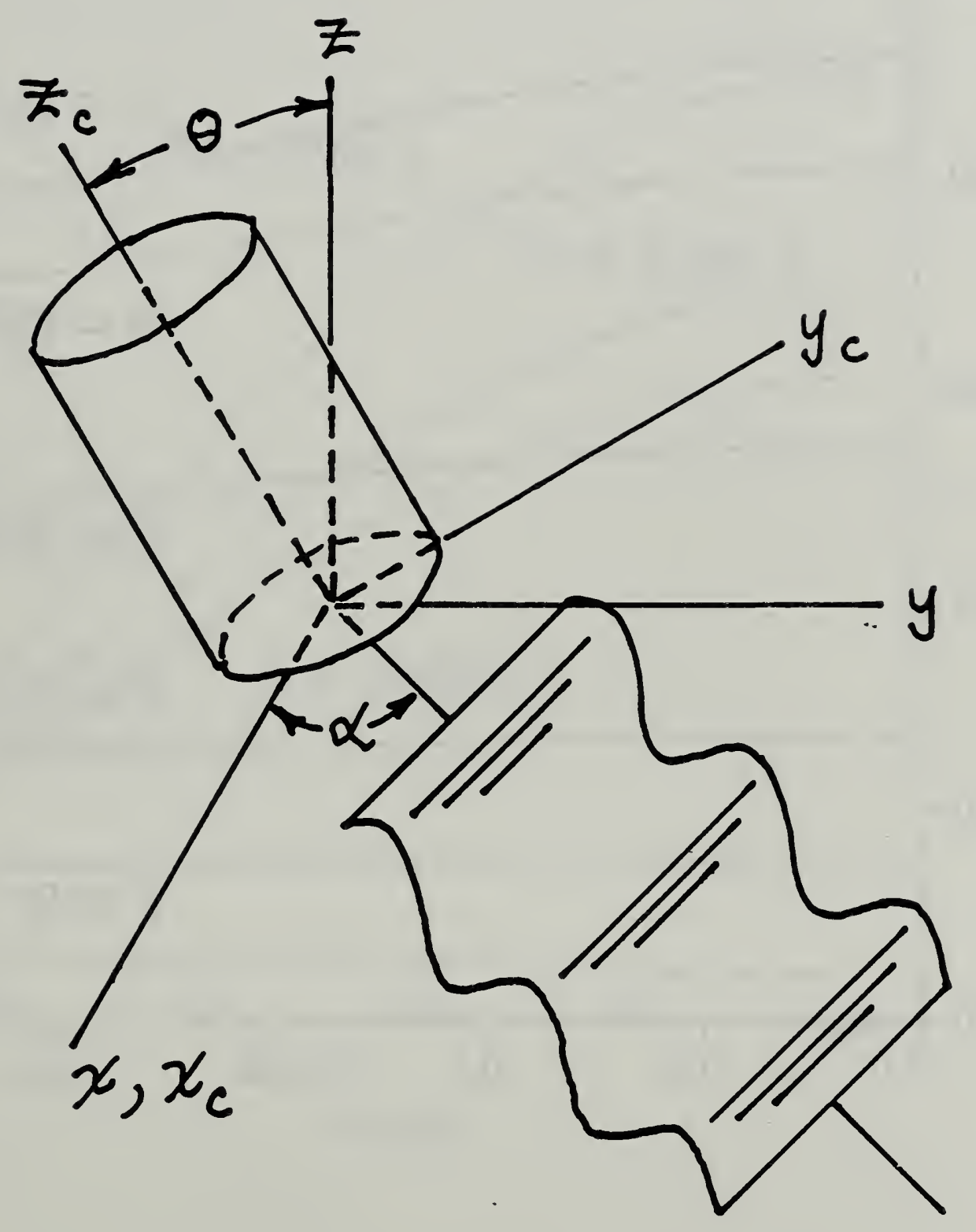

Fig. 1 


\section{Morphological Stability Tin-Bismuth $\mathrm{C}_{\infty}=0.78$ at $\% \quad \mathrm{~V}=5 \mu \mathrm{m} / \mathrm{s}$}

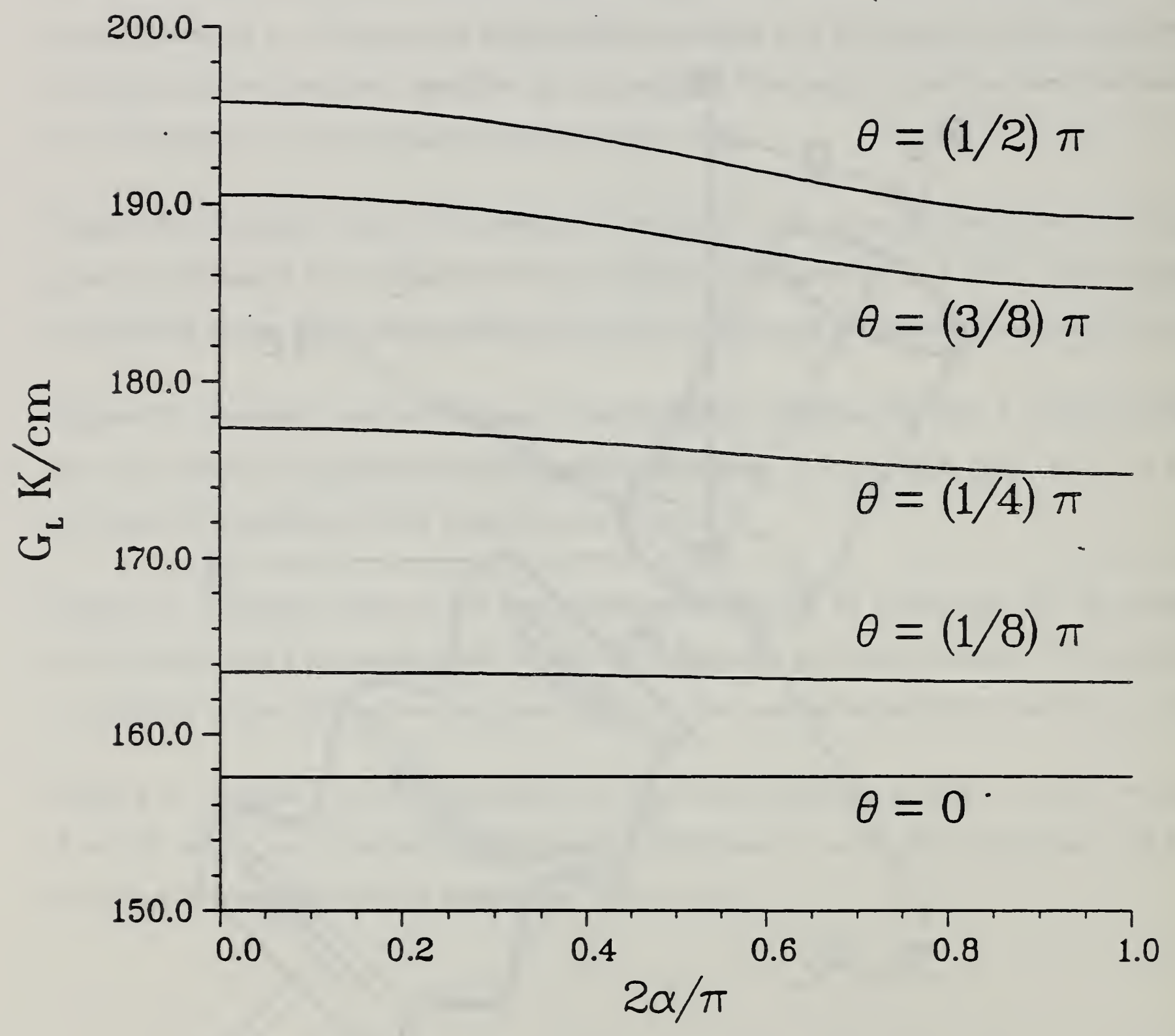




\section{Morphological Stability Tin-Bismuth $\mathrm{C}_{\infty}=0.78$ at $\% \quad \mathrm{~V}=5 \mu \mathrm{m} / \mathrm{s}$}

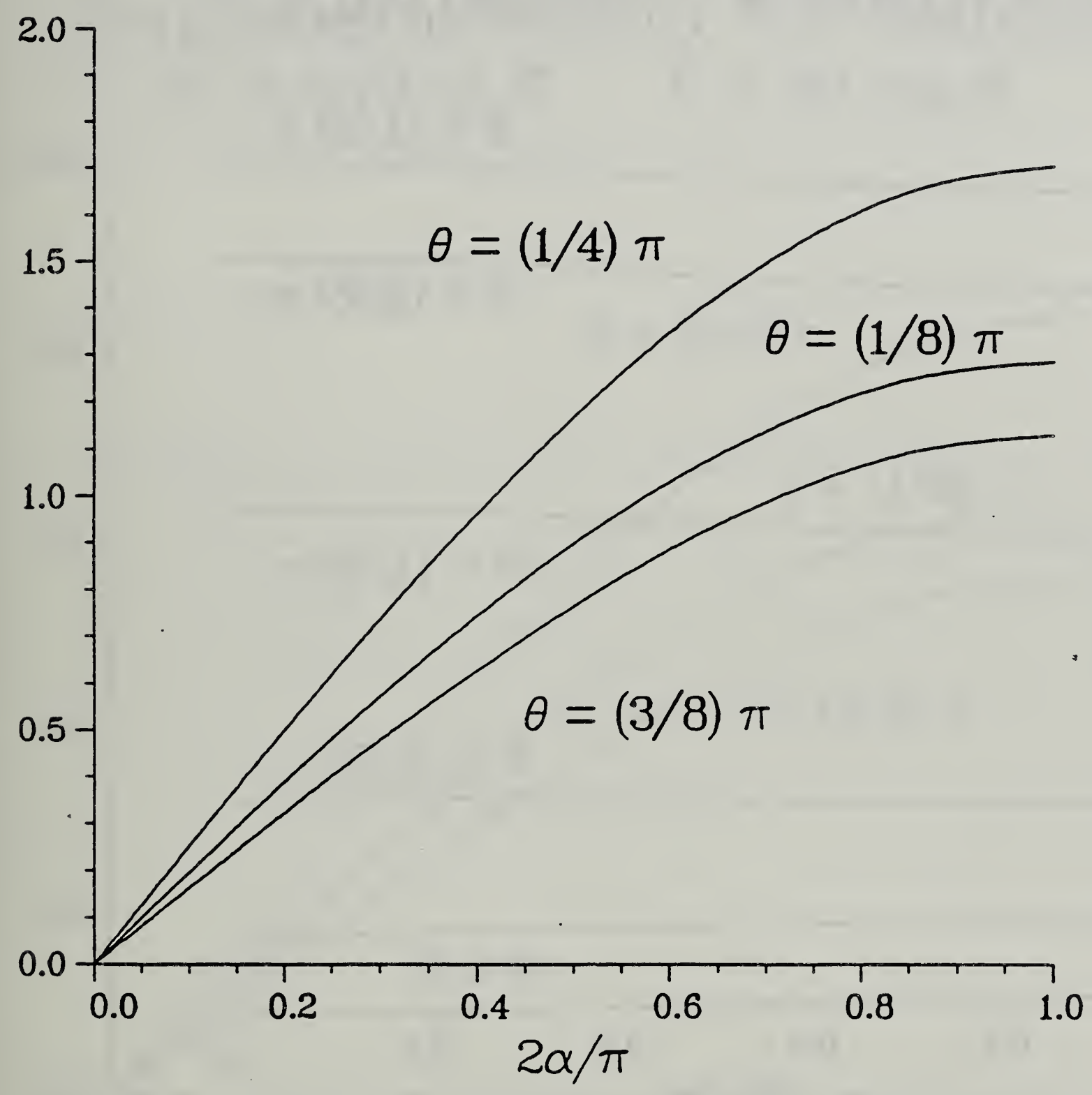


Morphological Stability Tin-Bismuth

$$
\mathrm{C}_{\infty}=0.78 \text { at } \% \quad \mathrm{~V}=30 \mathrm{~cm} / \mathrm{s}
$$

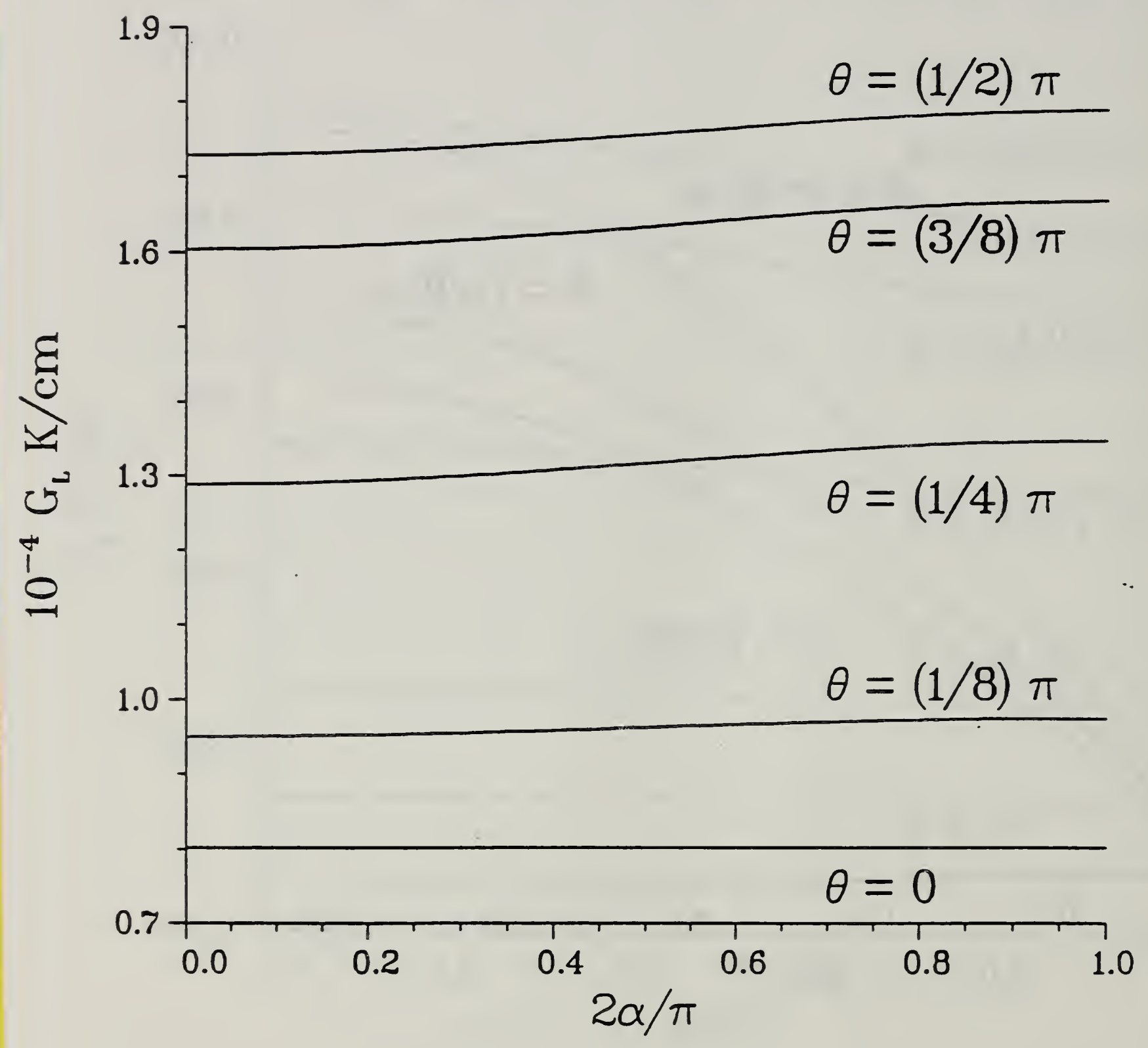




\section{Morphological Stability Tin-Bismuth $\mathrm{C}_{\infty}=0.78$ at $\% \quad \mathrm{~V}=30 \mathrm{~cm} / \mathrm{s}$}

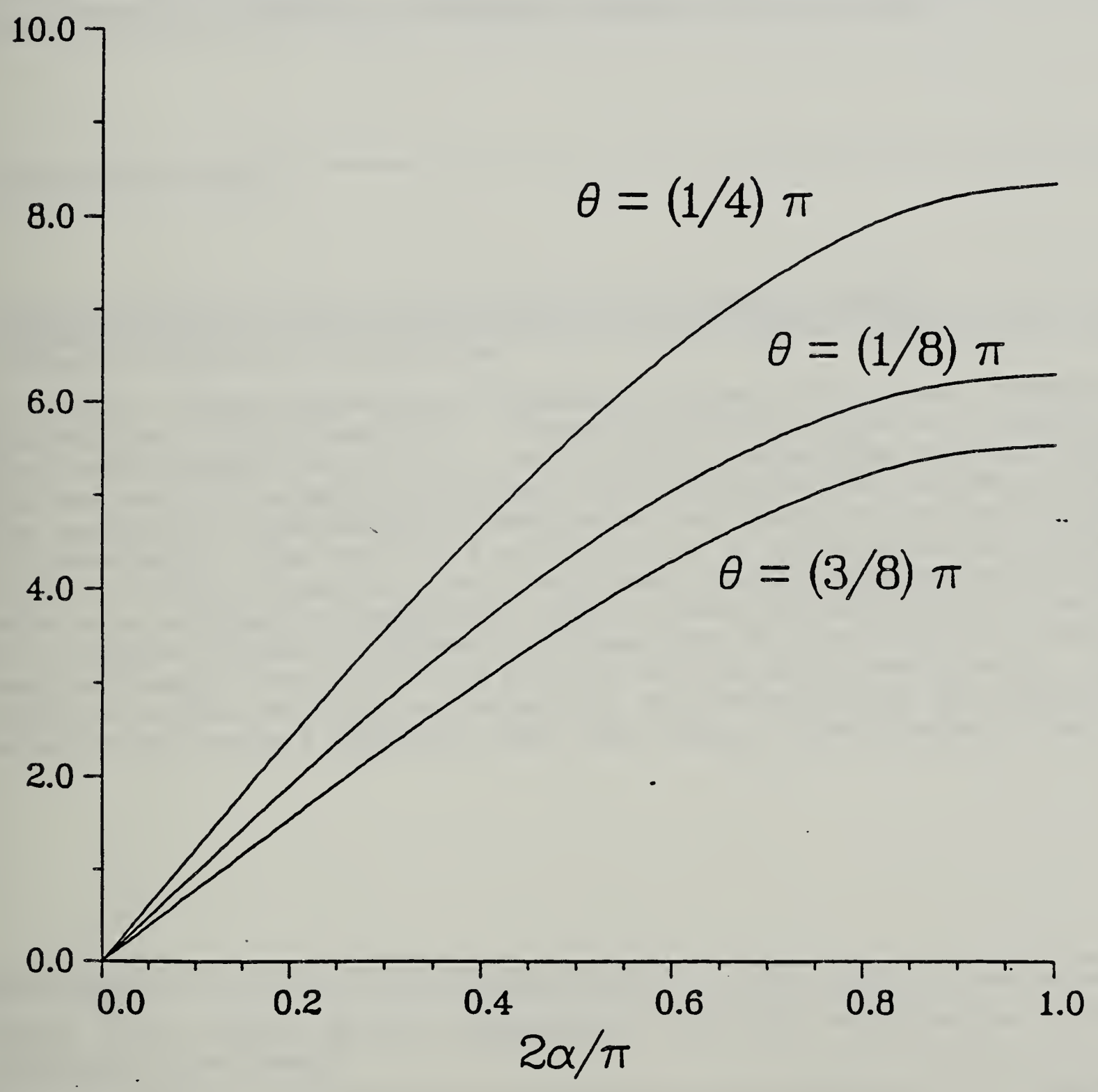

Fig. 5 

NOJ-1 IAA IREV. LOOWI

U.S. DEPT. OF COMM.

PUBLICATION OR

REPORT NO.

NISTIR $89-4143$

BIBLIOGRAPHIC DATA

SHEET (See in structions)

4. TITLE AND SUBTITLE

Effect of Anisotropic Thermal Conductivity on the

Morphological Stability of a Binary Alloy

5. $A U T H O R(S)$

G. B. McFadden, S. R. Coriell and R. F. Sekerka

6. PERFORMING ORGANIZATION (If joint or other than NBS, see instructions)

7. Contract/Grant No.

NATIONAL BUREAU OF STANDARDS

U.S. DEPARTMENT OF COMMERCE

GAITHERSBURG, MD 20899

9. SPONSORING ORGANIZATION NAME AND COMPLETE ADDRESS (Street, City, Stote, ZIP)

8. Type of Report \& Period Covered

10. SUPPLEMENTARY NOTES

Document describes a computer program; SF-185, FIPS Software Summary, is attached.

11. ABSTRACT (A 200-word or less factual summary of most significant information. If document includes a significant bibliogrophy or literature survey. mention it here)

We perform a linear morphological stability analysis of a planar interface during unidirectional solidification of a binary alloy for the case of a crystal having an anisotropic thermal conductivity. We calculate a dispersion relation which shows that the onset of instability depends on the orientation of the growth direction with respect to crystallographic axes and on the orientation of the wayevector of the perturbation. The onset of instability can be either oscillatory (travelling waves) or non-oscillatory in time. For growth along a principal axis of the crystal there is an exchange of stabilities, and the onset of instability is non-oscillatory. For a uniaxial crystal we explore the dispersion relation in detail, and give numerical results for the case of an alloy of 0.78 at $\%$ bismuth in tin. For low growth velocities the onset of instability is non-oscillatory and occurs for perturbations having a wavevector that lies along a crystallographic axis.

12. KEY WORDS (Six to twelve entries: alphabetical order: capitalize only proper names; and separate key words by semicolons) binary alloy; crystal growth; morphological stability; solification; thermal anisotropy; Tin-Bismuth

\section{AVAILABILITY}

Unlimited

For Official Distribution. Do Not Release to NTIS

$\square$ Order From Superintendent of Documents, U.S. Government Printing Office, Washington. D.C. 20402.

X Order From National Technical Information Service (NTIS), Springfield, VA. 2216I
14. NO. OF

PRINTED PAGES

23

15. Prire

$\$ 9.95$ 


IR 89-4144

\section{RESTRICTED}


4614 $x=1$

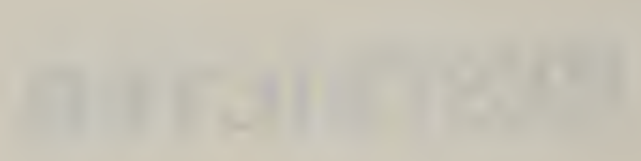

